\title{
The hypoxic microenvironment of stem cells and their progenies of the heart
}

\begin{abstract}
Ischemic cardiovascular disease, including myocardial infarction and heart failure, is among the primary causes of death worldwide. Therapies utilizing stem cells for cardiovascular regeneration in humans represent promising strategies to treat cardiovascular disease. In the adult heart, the hypoxic microenvironment typical of pathological conditions such as heart failure and myocardial infarction triggers the transcription of a series of genes involved in the regulation of deleterious effects caused by oxygen insufficiency at the cellular, tissue, and systemic levels. During embryonic cardiovascular development, variations in oxygen content within the stem cell microenvironment play a major role in the regulation of a diverse range of cellular processes, such as glycolysis, angiogenesis, apoptosis and cell proliferation. This review will provide an overview of the roles played by the hypoxic microenvironment on stem cells and their progenies of the heart in vivo and in vitro for optimal designing of the microenvironment for regenerative studies in humans
\end{abstract}

Keywords: hypoxia, cardiovascular disease, regenerative therapy, stem cells, hypoxia inducible factors, microenvironment
Volume 2 Issue 6 - 2017

\author{
Poonam Sharma, Gemma Figtree, Carmine \\ Gentile \\ University of Sydney, Australia
}

Correspondence: Carmine Gentile, Sydney Medical Schoo (Northern), University of Sydney, Sydney, NSW, Australia, Tel +6|299264937, Email carmine.gentile@sydney.edu.au

Received: May 27, 2017 | Published: June 08, 2017

\section{Introduction}

Cardiovascular disease is a major cause of lifelong disability and the most common form of heart disease is ischaemic heart disease, characterized by an imbalance between myocardial oxygen supply and its demand. This can lead to disturbances in impulse formation and conduction in the heart in the form of arrhythmias. During myocardial infarction, a sustained ischaemia leads to irreversible necrosis of the heart muscle and a plethora of stem cell therapies have been investigated to treat cardiovascular disease. ${ }^{1,2}$ Several studies testing the regenerative potential of stem cells to treat cardiovascular disease in humans have focused on designing the optimal microenvironment for these cells. ${ }^{3}$ Based on their potency, stem cells of the heart can be divided as: i) totipotent; ii) pluripotent, such as "embryonic stem cells" ("ESCs") and "induced-pluripotent stem cells" ("iPSCs"); iii) multipotent, such as "hematopoietic stem cells" ("HSCs") and "mesenchymal stem cells" ("MSCs"); and iv) unipotent, such as "cardiac stem cells" ("CSCs"). ${ }^{3}$ Lastly, other progenitor cells of the heart can be identified, such as "skeletal myoblasts" (also referred to as "skeletal muscle satellite cells") and "mesodermal VEGFR2 ${ }^{+}$cardiac progenitor cells". ${ }^{3}$ For regenerative therapy purposes, several stem cell types and progenies at different developmental stages have been considered for transplantation into the ischemic and hold promise for future studies aiming at regenerate the heart in cardiovascular disease patients as described in this review.

\section{Oxygen content in the stem cell microenvi- ronment}

During hypoxia, a number of adaptive physiological responses occur that regulate metabolism, redox homeostasis and vascular remodeling. ${ }^{4}$ Hypoxic conditions in the adult heart is predominantly associated with pathological conditions, such as heart failure and myocardial infarction, whereas embryonic heart development is characterized by a physiological regulation of the oxygen content in the stem cell microenvironment. ${ }^{3}$ Hypoxia may trigger the transcription of a large number of genes involved in a variety of cellular processes such as glycolysis, angiogenesis, apoptosis and cell proliferation, aiming at minimizing deleterious effects caused by oxygen insufficiency at the cellular, tissue, and systemic level. Hypoxia has been linked to stem cell quiescence, whereas normoxia is thought to be required for stem cell activation. However, a complete understanding of the regulation of the stem cell microenviroment by normoxic/hypoxic conditions during development has to be defined yet. ${ }^{5}$

\section{Role of hifs in hypoxic microenvironment}

Hypoxia inducible factors (HIFs) are transcription factors which belong to the basic helix-loop-helix-Per-Arnt-Sim family and play a major role in the regulation of the heart microenvironment following changes in oxygen content. ${ }^{3,6}$ HIFs are heterodimeric protein complexes consisting of an oxygen-independent $\beta$ subunit and an oxygen-dependent $\alpha$ subunit with tightly regulated protein stability and activity. Three HIF- $\alpha$ factors-HIF- $1 \alpha$, HIF- $2 \alpha$ and HIF$3 \alpha$ have been recognized. ${ }^{6}$ HIF- $1 \alpha$ and HIF- $2 \alpha$ are closely related in their protein structures and both recognize common HIF responsive elements, but they differ in their tissue expression profiles. HIF-1 $\alpha$ is expressed ubiquitously under hypoxic conditions in the heart and it regulates the anaerobic glycolytic metabolism in the cytoplasm. HIF- $2 \alpha$ is expressed in vascular endothelial cells during embryonic development, whereas in the adult it is expressed throughout the heart, regulating its oxidative stress response. ${ }^{7} \mathrm{HIF}-3 \alpha$ function has not been defined yet, but it may act as a negative regulator of HIF- $1 \alpha$ and HIF$2 \alpha .^{7}$ Oxygen concentration of the microenvironment tightly regulates HIF activity at the transcriptional, translational and posttranscriptional levels. In normoxic conditions, hydroxylation of HIF $1 \alpha$ subunits via three prolyl hydroxylase domain-containing enzymes (PHD1-3) are responsible for HIF $1 \alpha$ degradation, which is reverted in hypoxic conditions. $^{8}$ 


\section{Effect of oxygen content on in vitro stem cell differentiation}

To date, no in vitro model has fully recreated the oxygen content and its variation over time typical of the microenvironment of all cardiac stem cells. However, several studies have focused on recreating the in vivo hypoxic conditions for each stem cell type based on their origin and application. ${ }^{3}$ For instance, hypoxia does not regulate human ESC proliferation, but it has been shown to be critical for their differentiation into cardiac cells, mainly triggered under hypoxic conditions. ${ }^{5}$ Yang et al., ${ }^{6}$ showed that differentiation of myoblasts is favoured in a hypoxic microenvironment, with undifferentiated myoblasts presenting high levels of HIF $1 \alpha$, whereas differentiated myoblasts express high levels of HIF $2 \alpha$. The study by Kudová et al., ${ }^{9}$ showed that HIF-1 $\alpha$ deficiency significantly impaired the transcription of cardiac cell specific genes, and therefore the differentiation of ESCs into cardiomyocytes. The same study demonstrated that ESCs and iPSCs adapt differently to the hypoxic environment compared with their progenies. The study by Abaci et al., ${ }^{10}$ demonstrated that ESCs and iPSCs consume comparable amount of oxygen regardless of the amount present in their microenvironment, whereas human endothelial progenitor cells (hEPCs), and human umbilical cord vein endothelial cells (HUVECs) adapt their oxygen consumption depending on the oxygen availability.

\section{Molecular regulation of stem cell behavior in the heart}

From the molecular point of view, the different response to oxygen content between ESCs/iPSCs and hEPCs/HUVECs has been linked to overexpression of angiopietin1 (ANGPT1) in ESCs and iPSCs in hypoxic conditions. ${ }^{10}$ This has been demonstrated to be a HIF-1 $\alpha$ -independent mechanism. ${ }^{11}$ On the other side, Cardiac Stem Cells (CSCs) have been shown to play a protective role against hypoxiainduced injury in vivo via an MCP-1-dependent mechanism. ${ }^{11}$ MSC adhesion, migration, and proliferation via SUMO and Notch pathways in a HIF $1 \alpha$-dependent mechanism. ${ }^{12}$ The ERK signaling pathway has been shown to play a major role in regulating hypoxiainduced cardiac progenitor cell differentiation into cardiomyocytes. ${ }^{13}$ More recently, Nakada et al., ${ }^{14}$ have demonstrated that hypoxia induces heart regeneration in adult mice by promoting cardiomyocyte mitosis and preventing oxidative stress-derived myocardial damage. However, further studies are required to completely understand the signaling pathways involved in this mechanism.

\section{Conclusion}

In this review, we have highlighted some of the major roles played by the oxygen content typical of the microenvironment on both fate and behavior of stem cells and their progenies in the heart (Figure 1). Hypoxic conditions induce differentiation of most stem cell types at the transcriptional level of cardiac genes, via either a HIF$1 \alpha$-dependent or HIF-1 $\alpha$-independent pathway. However, lack of optimal models of the stem cell microenvironment of the human heart has limited our understanding of how oxygen content can regulate cardiac regeneration. Further studies generating models that better recapitulate the oxygen content typical of a developing human heart have the potential to provide insights for stem cell therapies aiming at the regeneration of the heart of cardiovascular disease patients.

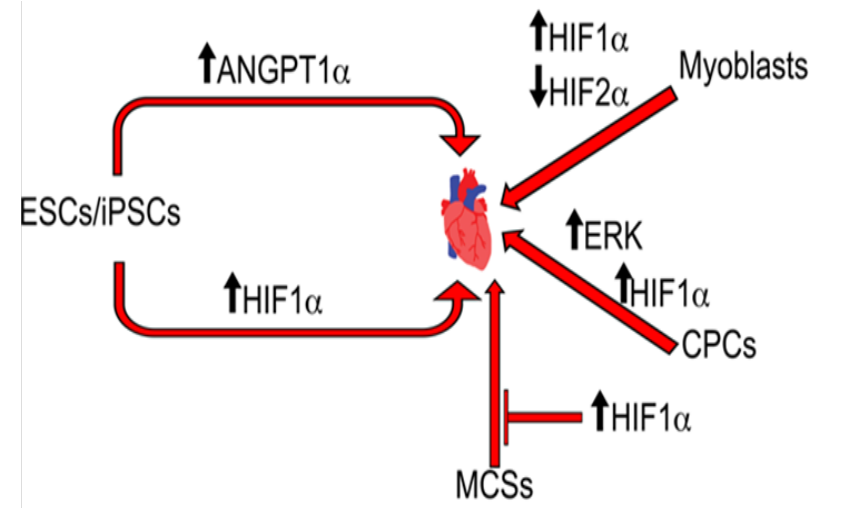

Figure I Signalling pathways regulating hypoxia-driven differentiation of stem cells and their progenies in the heart. Schematic showing some of the main signaling pathways regulating differentiation of stem cells and their progenies towards lineages of the human heart.

\section{Acknowledgements}

This study was supported by the Kick-Start Grant from the University of Sydney to CG and by an NHMRC Project Grant (APP1129685) to GF and CG. GF is supported by an NHMRC Career Development Fellowship and a National Heart Foundation Future Leader Fellowship. GF is supported by Heart Research Australia.

\section{Conflict of interest}

The author declares no conflict of interest.

\section{References}

1. Ji ST, Kim H, Yun J, et al. Promising therapeutic strategies for mesenchymal stem cell-based cardiovascular regeneration: From cell priming to tissue engineering. Stem Cells Int. 2017;2017:3945403.

2. Dixit P, Katare R. Challenges in identifying the best source of stem cells for cardiac regeneration therapy. Stem Cell Res Ther. 2015;13(6):26.

3. Gentile C. Filling the Gaps between the in vivo and in vitro microenvironment: engineering of spheroids for stem cell technology. Curr Stem Cell Res Ther. 2016;11(8):652-665.

4. Lahiri S, Roy A, Baby SM, et al. Oxygen sensing in the body. Prog Biophys Mol Biol. 2006;91(3):249-286.

5. Roberts JE, Wielgus AR, Boyes WK, et al. Phototoxicity and cytotoxicity of fullerol in human lens epithelial cells. Toxicol Appl Pharmacol. 2008;228(1):49-58.

6. Yang X, Yang S, Wang C, et al. The hypoxia-inducible factors HIF1alpha and HIF2alpha are dispensable for embryonic muscle development but essential for postnatal muscle regeneration. J Biol Chem. 2017;292(14):5981-5991.

7. Yang $\mathrm{C}, \mathrm{Xu} \mathrm{Y}, \mathrm{Yu} \mathrm{M}$, et al. Induced pluripotent stem cell modelling of HLHS underlines the contribution of dysfunctional NOTCH signalling to impaired cardiogenesis. Hum Mol Genet. 2017;26(16):3031-3045.

8. Bertout JA, Patel SA, Simon MC. The impact of $\mathrm{O}_{2}$ availability on human cancer. Nat Rev Cancer. 2008;8(12):967-975.

9. Jana K, Jiřina P, Ondřej V, et al. HIF-1alpha Deficiency Attenuates the Cardiomyogenesis of Mouse Embryonic Stem Cells. PLoS One. 2016;11(6):e0158358. 
10. Abaci HE, Truitt R, Luong E, et al. Adaptation to oxygen deprivation in cultures of human pluripotent stem cells, endothelial progenitor ce1ls, and umbilical vein endothelial cells. Am J Physiol Cell Physiol. 2010;298(6):1527-1537.

11. Park CY, Choi SC, Kim JH, et al. Cardiac stem cell secretome protects cardiomyocytes from hypoxic Injury partly via monocyte chemotactic protein-1-dependent mechanism. Int J Mol Sci. 2016;17(6):E800.

12. Ciria M, García NA, OntoriaO, et al. Mesenchymal stem cell migration and proliferation are mediated by hypoxia-inducible factor -1alpha upstream of notch and SUMO pathways. Stem Cells Dev. 2017;26(13):973-985.
13. Jung SY, Choi SH, Yoo SY, et al. Modulation of human cardiac progenitors via hypoxia-erk circuit improves their functional bioactivities. Biomol Ther (Seoul). 2013;21(3):196-203.

14. Nakada Y, Canseco DC, Thet S, et al. Hypoxia induces heart regeneration in adult mice. Nature. 2017;541(7636):222-227. 